Journal of Systems Science and Information

Feb., 2016, Vol. 4, No. 1, pp. 87-96

DOI: $10.1515 /$ JSSI-2016-0087

\title{
Smoothing Approximation to the Square-Root Exact Penalty Function
}

\author{
Yaqiong DUAN \\ College of Management, Qufu Normal University, Rizhao 276826, China \\ E-mail: 287093065@qq.com \\ Shujun LIAN* \\ College of Management, Qufu Normal University, Rizhao 276826, China \\ E-mail: lsjsd2003@126.com
}

\begin{abstract}
In this paper, smoothing approximation to the square-root exact penalty functions is devised for inequality constrained optimization. It is shown that an approximately optimal solution of the smoothed penalty problem is an approximately optimal solution of the original problem. An algorithm based on the new smoothed penalty functions is proposed and shown to be convergent under mild conditions. Three numerical examples show that the algorithm is efficient.
\end{abstract}

Keywords constrained optimization; exact penalty function; square-root penalty function; optimal solution; smoothing method

\section{Introduction}

Consider the following nonlinear constrained optimization problem

$$
\begin{aligned}
& \min f(x) \\
{[\mathrm{P}] \quad \text { s.t. } } & g_{i}(x) \leq 0, \quad i=1,2, \cdots, m, \\
& x \in R^{n},
\end{aligned}
$$

where $f: R^{n} \rightarrow R$ and $g_{i}: R^{n} \rightarrow R, i \in I=\{1,2, \cdots, m\}$ are twice continuously differentiable functions. Let

$$
G_{0}=\left\{x \in R^{n} \mid g_{i}(x) \leq 0, \quad i=1,2, \cdots, m\right\}
$$

To solve $[\mathrm{P}]$, many penalty function methods have been proposed in the literatures (see, e.g., [1-10]). In [1] the classical $l_{1}$ exact penalty function is defined as follows

$$
f(x, q)=f(x)+q \sum_{i=1}^{m} g_{i}^{+}(x),
$$

where $g_{i}^{+}(x)=\max \left\{0, g_{i}(x)\right\}, i=1,2, \cdots, m$.

Received September 14, 2015, accepted October 16, 2015

Supported by National Natural Science Foundation of China (71371107 and 61373027) and Natural Science Foundation of Shandong Provence (ZR2013AM013 and ZR2012AL07)

${ }^{*}$ The corresponding author 
Nonlinear penalty function has been investigated in [11] and [12] as the following form

$$
L^{k}(x, d)=\left[f(x)^{k}+\sum_{i=1}^{m} d_{i}\left(g_{i}^{+}(x)\right)^{k}\right]^{1 / k},
$$

where $f(x)$ is assumed to be positive, $k>0$ is a given number, and $d=\left(d_{1}, d_{2}, \cdots, d_{m}\right) \in R_{+}^{m}$ is the penalty parameter. In [11], it was shown that the exact penalty parameter corresponding to $k \in(0,1]$ is substantially smaller than that of the classical $l_{1}$ exact penalty function.

In [13], the lower order exact penalty functions

$$
\varphi_{q, k}(x)=f(x)+q \sum_{i=1}^{m}\left(g_{i}^{+}(x)\right)^{k}, \quad k \in(0,1)
$$

have been investigated. It is shown that any strict local minimizer satisfying the second order sufficiency condition for the original problem is a strict local minimizer of the lower order penalty function with any positive penalty parameter. However, it is not a smooth function. When $k=\frac{1}{2}$, smoothing for the nonlinear penalty function

$$
\varphi_{q}(x)=f(x)+q \sum_{i=1}^{m} \sqrt{g_{i}^{+}(x)}
$$

was investigated in [14] and [15].

In this paper, we propose a method for smoothing the square-root penalty function of the form (2). Different from the smooth functions given in [14] and [15], we give a function approximate to the original function from the left side of 0 . The rest of this paper is organized as follows. In Section 2, a new smoothing function to the square-root penalty function is introduced. It is shown that an approximately optimal solution of the smoothed penalty problem is an approximately optimal solution of the original problem. In Section 3, we give an algorithm to compute an approximate solution to $[\mathrm{P}]$ based on the smooth penalty function and show that the algorithm is convergent. In Section 4, three numerical examples are given to show the efficiency of the algorithm.

\section{Smoothing Exact Lower Order Penalty Function}

Consider the following lower order penalty problem

$$
\text { [LOP] } \min _{x \in R^{n}} \varphi_{q}(x) .
$$

In order to establish the exact penalization, we need the following assumptions given in [13].

Assumption $1 \quad f(x)$ satisfies the following coercive condition

$$
\lim _{\|x\| \rightarrow+\infty} f(x)=+\infty .
$$

Under Assumption 1, there exists a box $X$ such that $G([\mathrm{P}]) \subset \operatorname{int}(X)$, where $G([\mathrm{P}])$ is the set of global minima of problem $[\mathrm{P}], \operatorname{int}(X)$ denotes the interior of the set $X$. Consider the following problem

$$
\begin{aligned}
& \min f(x) \\
{\left[\mathrm{P}^{\prime}\right] \quad \text { s.t. } } & g_{i}(x) \leq 0, \quad i=1,2, \cdots, m, \\
& x \in X
\end{aligned}
$$


Let $G\left(\left[\mathrm{P}^{\prime}\right]\right)$ denote the set of global minima of problem $\left[\mathrm{P}^{\prime}\right]$. Then $G\left(\left[\mathrm{P}^{\prime}\right]\right)=G([\mathrm{P}])$.

Assumption 2 The set $G([\mathrm{P}])$ is a finite set.

Then we consider the penalty problem of the form

$$
\left[\mathrm{LOP}^{\prime}\right] \min _{x \in X} \varphi_{q}(x)
$$

Let $p(u)=(\max \{0, u\})^{\frac{1}{2}}$, that is,

$$
p(u)= \begin{cases}u^{\frac{1}{2}}, & \text { if } u>0 \\ 0, & \text { otherwise }\end{cases}
$$

then

$$
\varphi_{q}(x)=f(x)+q \sum_{i=1}^{m} p\left(g_{i}(x)\right)
$$

For any $\epsilon>0$, let

$$
p_{\epsilon}(u)= \begin{cases}\frac{2}{3} \epsilon^{\frac{1}{2}}, & \text { if } u \leq 0 \\ \frac{1}{3} \epsilon^{-1} u^{\frac{3}{2}}+\frac{2}{3} \epsilon^{\frac{1}{2}}, & \text { if } 0<u \leq \epsilon \\ u^{\frac{1}{2}}, & \text { if } u>\epsilon .\end{cases}
$$

It follows that

$$
p_{\epsilon}^{\prime}(u)= \begin{cases}0, & \text { if } u \leq 0 \\ \frac{1}{2} \epsilon^{-1} u^{\frac{1}{2}}, & \text { if } 0<u \leq \epsilon \\ \frac{1}{2} u^{-\frac{1}{2}}, & \text { if } u>\epsilon .\end{cases}
$$

It is easy to see that $p_{\epsilon}(u)$ is continuously differentiable on $R$. Furthermore, we can obtain that $p_{\epsilon}(u) \rightarrow p(u)$ as $\epsilon \rightarrow 0$.

Figure 1 shows the behavior of $p(u)$ (represented by the solid line), $p_{0.1}(u)$ (represented by the dot line), $p_{0.01}(u)$ (represented by the broken line) and $p_{0.001}(u)$ (represented by the dash and dot line).

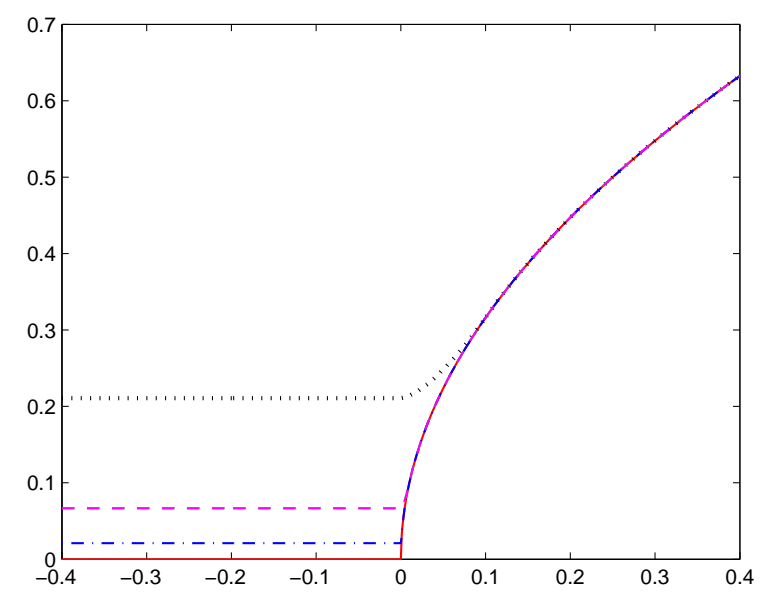

Figure 1 The behavior of $p_{\epsilon}(u)$ and $p(u)$ 
Let

$$
\varphi_{q, \epsilon}(x)=f(x)+q \sum_{i=1}^{m} p_{\epsilon}\left(g_{i}(x)\right) .
$$

Then $\varphi_{q, \epsilon}(x)$ is continuously differentiable on $R^{n}$. Consider the following smoothed optimization problem

$$
[\mathrm{SP}] \min _{x \in X} \varphi_{q, \epsilon}(x) .
$$

Lemma 1 For any $x \in X, \epsilon>0$,

$$
0 \leq \varphi_{q, \epsilon}(x)-\varphi_{q}(x) \leq \frac{2}{3} m q \epsilon^{\frac{1}{2}} .
$$

Proof Note that

$$
p_{\epsilon}\left(g_{i}(x)\right)-p\left(g_{i}(x)\right)= \begin{cases}\frac{2}{3} \epsilon^{\frac{1}{2}}, & \text { if } g_{i}(x) \leq 0, \\ -\left(g_{i}(x)\right)^{\frac{1}{2}}+\frac{1}{3} \epsilon^{-1}\left(g_{i}(x)\right)^{\frac{3}{2}}+\frac{2}{3} \epsilon^{\frac{1}{2}}, & \text { if } 0<g_{i}(x) \leq \epsilon, \\ 0, & \text { if } g_{i}(x)>\epsilon .\end{cases}
$$

When $g_{i}(x) \in(0, \epsilon]$, let

$$
F(u)=-u^{\frac{1}{2}}+\frac{1}{3} \epsilon^{-1} u^{\frac{3}{2}}+\frac{2}{3} \epsilon^{\frac{1}{2}}
$$

since

$$
F^{\prime}(u)=-\frac{1}{2} u^{-\frac{1}{2}}+\frac{1}{2} \epsilon^{-1} u^{\frac{1}{2}}=\frac{1}{2} \epsilon^{-1} u^{-\frac{1}{2}}(u-\epsilon)<0,
$$

we have

$$
0 \leq p_{\epsilon}\left(g_{i}(x)\right)-p\left(g_{i}(x)\right) \leq \frac{2}{3} \epsilon^{\frac{1}{2}}
$$

Then

$$
0 \leq \varphi_{q, \epsilon}(x)-\varphi_{q}(x) \leq \frac{2}{3} m q \epsilon^{\frac{1}{2}} .
$$

This completes the proof.

Theorem 2 Let $\left\{\epsilon_{j}\right\} \rightarrow 0$ be a sequence of positive numbers and assume that $x^{j}$ is a solution to $\min _{x \in X} \varphi_{q, \epsilon_{j}}(x)$ for some $q>0$. Let $\bar{x}$ be an accumulating point of the sequence $\left\{x^{j}\right\}$. Then $\bar{x}$ is an optimal solution to $\min _{x \in X} \varphi_{q}(x)$.

Proof Because $x^{j}$ is a solution to $\min _{x \in X} \varphi_{q, \epsilon_{j}}(x)$, we have

$$
\varphi_{q, \epsilon_{j}}\left(x^{j}\right) \leq \varphi_{q, \epsilon_{j}}(x), \quad \forall x \in X .
$$

By Lemma 1, we have

$$
\varphi_{q}\left(x^{j}\right) \leq \varphi_{q, \epsilon_{j}}\left(x^{j}\right)
$$

and

$$
\varphi_{q, \epsilon_{j}}(x) \leq \varphi_{q}(x)+\frac{2}{3} m q \epsilon_{j}^{\frac{1}{2}} .
$$

It follows that

$$
\varphi_{q}\left(x^{j}\right) \leq \varphi_{q, \epsilon_{j}}\left(x^{j}\right) \leq \varphi_{q, \epsilon_{j}}(x) \leq \varphi_{q}(x)+\frac{2}{3} m q \epsilon_{j}^{\frac{1}{2}} .
$$

Let $j \rightarrow 0$, we have

$$
\varphi_{q}(\bar{x}) \leq \varphi_{q}(x)
$$


Theorem 3 Let $x_{q}^{*} \in X$ be an optimal solution of problem $\left[\mathrm{LOP}^{\prime}\right]$ and $\bar{x}_{q, \epsilon} \in X$ be an optimal solution of problem [SP] for some $q>0$ and $\epsilon>0$. Then

$$
0 \leq \varphi_{q, \epsilon}\left(\bar{x}_{q, \epsilon}\right)-\varphi_{q}\left(x_{q}^{*}\right) \leq \frac{2}{3} m q \epsilon^{\frac{1}{2}}
$$

Proof By Lemma 1, we have

$$
\begin{aligned}
0 \leq \varphi_{q, \epsilon}\left(\bar{x}_{q, \epsilon}\right)-\varphi_{q}\left(\bar{x}_{q, \epsilon}\right) & \leq \varphi_{q, \epsilon}\left(\bar{x}_{q, \epsilon}\right)-\varphi_{q}\left(x_{q}^{*}\right) \\
& \leq \varphi_{q, \epsilon}\left(x_{q}^{*}\right)-\varphi_{q}\left(x_{q}^{*}\right) \\
& \leq \frac{2}{3} m q \epsilon^{\frac{1}{2}}
\end{aligned}
$$

Corollary 4 Suppose that Assumptions 1 and 2 hold, and that for any $x^{*} \in G([\mathrm{P}])$, there exists a $\lambda^{*} \in R_{+}^{m}$ such that the pair $\left(x^{*}, \lambda^{*}\right)$ satisfies the second order sufficiency condition defined in [2]. Let $x^{*} \in X$ be a global solution of problem $[\mathrm{P}]$ and $\bar{x}_{q, \epsilon} \in X$ be a global solution of problem $[\mathrm{SP}]$ for $\epsilon>0$. Then there exists $q^{*}>0$ such that for any $q>q^{*}$,

$$
0 \leq \varphi_{q, \epsilon}\left(\bar{x}_{q, \epsilon}\right)-f\left(x^{*}\right) \leq \frac{2}{3} m q \epsilon^{\frac{1}{2}},
$$

where $q^{*}$ is defined in Corollary 2.3 in [13].

Proof By Corollary 2.3 in [13], we have that $x^{*}$ is a global solution of problem [LOP'] for $q>q^{*}$ for an appropriately chosen $q^{*}>0$. Then by Theorem 3 , we have

$$
0 \leq \varphi_{q, \epsilon}\left(\bar{x}_{q, \epsilon}\right)-\varphi_{q}\left(x^{*}\right) \leq \frac{2}{3} m q \epsilon^{\frac{1}{2}}
$$

Since $\sum_{i=1}^{m} p\left(g_{i}\left(x^{*}\right)\right)=0$, we have

$$
\varphi_{q}\left(x^{*}\right)=f\left(x^{*}\right)+q \sum_{i=1}^{m} p\left(g_{i}\left(x^{*}\right)\right)=f\left(x^{*}\right) .
$$

Definition 5 For $\epsilon>0$, a point $x \in X$ is said to be an $\epsilon$-feasible solution of problem [P], if $g_{i}(x) \leq \epsilon$ for any $i \in I$.

Theorem 6 Let $x_{q}^{*} \in X$ be an optimal solution of problem $\left[L O P^{\prime}\right]$ and $\bar{x}_{q, \epsilon} \in X$ be an optimal solution of problem $[\mathrm{SP}]$. Furthermore, let $x_{q}^{*}$ be a feasible solution of problem $[\mathrm{P}]$ and $\bar{x}_{q, \epsilon}$ be an $\epsilon$-feasible solution of problem $[\mathrm{P}]$, then we have

$$
-m q \epsilon^{\frac{1}{2}} \leq f\left(\bar{x}_{q, \epsilon}\right)-f\left(x_{q}^{*}\right) \leq 0 .
$$

Proof It is clear that $\sum_{i=1}^{m} p\left(g_{i}\left(x_{q}^{*}\right)\right)=0$. By Theorem 3 we have

$$
\begin{aligned}
0 & \leq \varphi_{q, \epsilon}\left(\bar{x}_{q, \epsilon}\right)-\varphi_{q}\left(x_{q}^{*}\right) \\
& =f\left(\bar{x}_{q, \epsilon}\right)+q \sum_{i=1}^{m} p_{\epsilon}\left(g_{i}\left(\bar{x}_{q, \epsilon}\right)\right)-\left(f\left(x_{q}^{*}\right)+q \sum_{i=1}^{m} p\left(g_{i}\left(x_{q}^{*}\right)\right)\right) \leq \frac{2}{3} m q \epsilon^{\frac{1}{2}} .
\end{aligned}
$$

which implies

$$
-q \sum_{i=1}^{m} p_{\epsilon}\left(g_{i}\left(\bar{x}_{q, \epsilon}\right)\right) \leq f\left(\bar{x}_{q, \epsilon}\right)-f\left(x_{q}^{*}\right) \leq \frac{2}{3} m q \epsilon^{\frac{1}{2}}-q \sum_{i=1}^{m} p_{\epsilon}\left(g_{i}\left(\bar{x}_{q, \epsilon}\right)\right) .
$$

By (3), we have

$$
\frac{2}{3} \epsilon^{\frac{1}{2}} \leq p_{\epsilon}\left(g_{i}\left(\bar{x}_{q, \epsilon}\right)\right) \leq \epsilon^{\frac{1}{2}} .
$$


Then it follows (4) and (5) that

$$
-m q \epsilon^{\frac{1}{2}} \leq f\left(\bar{x}_{q, \epsilon}\right)-f\left(x_{q}^{*}\right) \leq 0 .
$$

This completes the proof.

Theorem 2 and Theorem 3 mean that an approximate solution to [SP] is also an approximate solution to $\left[\mathrm{LOP}^{\prime}\right]$ when the error $\epsilon$ is sufficiently small. Furthermore, by Theorem 6 , an optimal solution to $[\mathrm{SP}]$ becomes an approximately optimal solution to $[\mathrm{P}]$ if the solution to $[\mathrm{SP}]$ is $\epsilon$ feasible.

\section{A Smoothing Method}

We propose the following algorithm to solve $[\mathrm{P}]$.

Algorithm 7 Step 1 Choose an initial point $x^{0}$. Given $\epsilon_{0}>0, q_{0}>0,0<\eta<1$, and $N>$ 1 , let $j=0$ and go to Step 2 .

Step 2 Use $x^{j}$ as the starting point to solve $\min _{x \in R^{n}} \varphi_{q_{j}, \epsilon_{j}}(x)$. Let $x_{j}^{*}$ be the optimal solution obtained. ( $x_{j}^{*}$ is obtained by a quasi-Newton method and a finite difference gradient). Go to Step 3.

Step 3 If $x_{j}^{*}$ is $\epsilon$-feasible to [P], then stop and we have obtained an approximately optimal solution $x_{j}^{*}$ of the original problem $[\mathrm{P}]$. Otherwise, let $q_{j+1}=N q_{j}, \epsilon_{j+1}=\eta \epsilon_{j}, x^{j+1}=x_{j}^{*}$, and $j=j+1$, then go to Step 2 .

Remark 8 From $0<\eta<1$ and $N>1$, we can easily obtain the sequence $\left\{\epsilon_{j}\right\}$ is decreasing to 0 and the sequence $\left\{q_{j}\right\}$ is increasing to $+\infty$ as $j \longrightarrow+\infty$.

Now we prove the convergence of the algorithm under mild conditions.

Theorem 9 Suppose that for any $q \in\left[q_{0},+\infty\right), \epsilon \in\left(0, \epsilon_{0}\right]$, the set

$$
\arg \min _{x \in R^{n}} \varphi_{q, \epsilon}(x) \neq \emptyset .
$$

Let $\left\{x_{j}^{*}\right\}$ be the sequence generated by Algorithm 7. If $\left\{x_{j}^{*}\right\}$ has limit point, then any limit point of $\left\{x_{j}^{*}\right\}$ is the solution of $[\mathrm{P}]$ for any $m \geq 3$.

Proof Let $\bar{x}$ be any limit point of $\left\{x_{j}^{*}\right\}$. Then there exists a natural number set $J \subseteq N$, such that $x_{j}^{*} \rightarrow \bar{x}, j \in J$. If we can prove that (i) $\bar{x} \in G_{0}$ and (ii) $f(\bar{x}) \leq \inf _{x \in G_{0}} f(x)$ hold, then $\bar{x}$ is the optimal solution of $[\mathrm{P}]$.

(i) Suppose to contrary that $\bar{x} \notin G_{0}$, then there exist $\delta_{0}>0, i_{0} \in I$ and a subset $J_{1} \subset J$ such that $g_{i_{0}}\left(x_{j}^{*}\right) \geq \delta_{0}$ for any $j \in J_{1}$.

If $\epsilon_{j} \geq g_{i_{0}}\left(x_{j}^{*}\right) \geq \delta_{0}$, it follows from Step 2 in Algorithm 7 and (3) that

$$
f\left(x_{j}^{*}\right)+\frac{1}{3} q_{j} \epsilon_{j}^{-1} \delta_{0}^{\frac{3}{2}}+\frac{2}{3} m q_{j} \epsilon_{j}^{\frac{1}{2}} \leq \varphi_{q_{j}, \epsilon_{j}}\left(x_{j}^{*}\right) \leq \varphi_{q_{j}, \epsilon_{j}}(x)=f(x)+\frac{2}{3} m q_{j} \epsilon_{j}^{\frac{1}{2}}, \quad \forall x \in G_{0} .
$$

Thus,

$$
f\left(x_{j}^{*}\right)+\frac{1}{3} q_{j} \epsilon_{j}^{-1} \delta_{0}^{\frac{3}{2}} \leq \varphi_{q_{j}, \epsilon_{j}}\left(x_{j}^{*}\right)-\frac{2}{3} m q_{j} \epsilon_{j}^{\frac{1}{2}} \leq f(x), \quad \forall x \in G_{0},
$$

which contradicts with $\epsilon_{j} \rightarrow 0$ and $q_{j} \rightarrow+\infty$.

If $g_{i_{0}}\left(x_{j}^{*}\right) \geq \delta_{0}>\epsilon_{j}$ or $g_{i_{0}}\left(x_{j}^{*}\right)>\epsilon_{j} \geq \delta_{0}$, it follows from Step 2 in Algorithm 7 and (3) that

$$
f\left(x_{j}^{*}\right)+q_{j} \delta_{0}^{\frac{1}{2}}+q_{j}(m-1) \epsilon_{j}^{\frac{1}{2}} \leq \varphi_{q_{j}, \epsilon_{j}}\left(x_{j}^{*}\right) \leq \varphi_{q_{j}, \epsilon_{j}}(x)=f(x)+\frac{2}{3} m q_{j} \epsilon_{j}^{\frac{1}{2}}, \quad \forall x \in G_{0} .
$$


Thus,

$$
f\left(x_{j}^{*}\right)+q_{j} \delta_{0}^{\frac{1}{2}}+\left(\frac{1}{3} m-1\right) q_{j} \epsilon_{j}^{\frac{1}{2}} \leq \varphi_{q_{j}, \epsilon_{j}}\left(x_{j}^{*}\right) \leq f(x), \quad \forall x \in G_{0},
$$

which contradicts with $\epsilon_{j} \rightarrow 0$ and $q_{j} \rightarrow+\infty$ when $m \geq 3$.

Then we have $\bar{x} \in G_{0}$.

(ii) For any $x \in G_{0}$, it holds that

$$
f\left(x_{j}^{*}\right) \leq \varphi_{q_{j}, \epsilon_{j}}\left(x_{j}^{*}\right) \leq \varphi_{q_{j}, \epsilon_{j}}(x)=f(x),
$$

then $f(\bar{x}) \leq \inf _{x \in G_{0}} f(x)$ holds. This completes the proof.

\section{Numerical Examples}

In this section, we solve three numerical examples to show the applicability of Algorithm 7 on Matlab.

Example 1 (Example 4.2 in [14] and Example 2 in [15])

$$
\begin{array}{cl}
\min & f(x)=x_{1}^{2}+x_{2}^{2}+2 x_{3}^{2}+x_{4}^{2}-5 x_{1}-5 x_{2}-21 x_{3}+7 x_{4} \\
\text { s.t. } & g_{1}(x)=2 x_{1}^{2}+x_{2}^{2}+x_{3}^{2}+2 x_{1}+x_{2}+x_{4}-5 \leq 0, \\
& g_{2}(x)=x_{1}^{2}+x_{2}^{2}+x_{3}^{2}+x_{4}^{2}+x_{1}-x_{2}+x_{3}-x_{4}-8 \leq 0, \\
& g_{3}(x)=x_{1}^{2}+2 x_{2}^{2}+x_{3}^{2}+2 x_{4}^{2}-x_{1}-x_{4}-10 \leq 0 .
\end{array}
$$

Let $x^{0}=(1,1,1,1), q_{0}=2.0, \epsilon_{0}=0.1, \eta=0.1, N=2$, the results by Algorithm 7 are shown in Table 1.

Table 1 Numerical results for Example 1

\begin{tabular}{cccccccc}
\hline$j$ & $x_{j}^{*}$ & $q_{j}$ & $\epsilon_{j}$ & $g_{1}\left(x_{j}^{*}\right)$ & $g_{2}\left(x_{j}^{*}\right)$ & $g_{3}\left(x_{j}^{*}\right)$ & $f\left(x_{j}^{*}\right)$ \\
\hline 0 & $\begin{array}{c}(1.138375,1.271269, \\
3.818479,-1.996515)\end{array}$ & 2 & 0.1 & 15.340209 & 19.160985 & 17.939222 & -70.152230 \\
\hline 1 & $\begin{array}{c}(0.177165,0.826666, \\
2.008946,-0.962929)\end{array}$ & 4 & 0.01 & 0.000079 & 0.000236 & -1.925763 & -44.233800 \\
\hline 2 & $\begin{array}{c}(0.177135,0.826640, \\
2.008904,-0.962939)\end{array}$ & 8 & 0.001 & -0.000241 & 0.000000 & -1.925947 & -44.233093 \\
\hline 3 & $\begin{array}{c}(0.177135,0.826640, \\
2.008903,-0.962938)\end{array}$ & 16 & 0.0001 & -0.000245 & -0.000006 & -1.925953 & -44.233076 \\
\hline
\end{tabular}

The obtained approximately optimal solution is $x^{*}=(0.1843219,0.8502275,1.992824$, $-0.9814662)$ with corresponding objective function value -44.233076 . From [14], the obtained approximately optimal solution is $x^{*}=(0.169234,0.835656,2.008690,-0.964901)$ with corresponding objective function value -44.233582 . From [15], the obtained approximately optimal solution is $x^{*}=(0.1585001,0.8339736,2.014753,-0.959688)$ with corresponding objective function value -44.22965 . 
For the $j^{\prime}$ th iteration of the algorithm, we define a constraint error $e_{j}$ by

$$
e_{j}=\sum_{i=1}^{m} \max \left(g_{i}\left(x_{j}^{*}\right), 0\right) .
$$

It is clear that $x_{j}^{*}$ is $\epsilon$-feasible to $(P)$ when $e_{j}<\epsilon$.

Example 2 (Example 4.3 in [14])

$$
\begin{array}{ll}
\min & f(x)=1000-x_{1}^{2}-2 x_{2}^{2}-x_{3}^{2}-x_{1} x_{2}-x_{1} x_{3} \\
\text { s.t. } & g_{1}(x)=x_{1}^{2}+x_{2}^{2}+x_{3}^{2}-25=0, \\
& g_{2}(x)=\left(x_{1}-5\right)^{2}+x_{2}^{2}+x_{3}^{2}-25=0, \\
& g_{3}(x)=\left(x_{1}-5\right)^{2}+\left(x_{2}-5\right)^{2}+\left(x_{3}-5\right)^{2}-25 \leq 0 .
\end{array}
$$

\begin{tabular}{|c|c|c|c|c|c|}
\hline$j$ & $x_{j}^{*}$ & $q_{j}$ & $\epsilon_{j}$ & $e_{j}$ & $f\left(x_{j}^{*}\right)$ \\
\hline 0 & $\left.\begin{array}{l}2.518662 \\
4.231055 \\
0.970488\end{array}\right)$ & 100 & 10 & 0.18804328 & 943.809922 \\
\hline 1 & $\left.\begin{array}{l}2.499999 \\
4.220526 \\
0.968073\end{array}\right)$ & 1000 & 0.1 & 0.000001 & 944.215680 \\
\hline 2 & $\left.\begin{array}{l}2.499999 \\
4.220525 \\
0.968073\end{array}\right)$ & 10000 & 0.001 & 0.000000 & 944.215671 \\
\hline
\end{tabular}

Starting point $x^{0}=(2,2,2), q_{0}=100, \epsilon_{0}=10, \eta=0.01, N=10$, we obtain the results by Algorithm 7 shown in Table 2 .

Table 2 Numerical results for Example 2

The results show that the obtained approximate global solution is $x_{j}^{*}=(2.499999,4.220525$, 0.968073 ) with objective function value $f\left(x_{j}^{*}\right)=944.215671$. From [14], we know that the global solution is $(2.500000,4.220720,0.967224)$ with global optimal value 944.215662 .

Example 3 (Example 4.5 in [14])

$$
\begin{array}{ll}
\min & f(x)=10 x_{2}+2 x_{3}+x_{4}+3 x_{5}+4 x_{6} \\
\text { s.t. } & g_{1}(x)=x_{1}+x_{2}-10=0, \\
& g_{2}(x)=-x_{1}+x_{3}+x_{4}+x_{5}=0, \\
& g_{3}(x)=-x_{2}-x_{3}+x_{5}+x_{6}=0, \\
& g_{4}(x)=10 x_{1}-2 x_{3}+3 x_{4}-2 x_{5}-16 \leq 0, \\
& g_{5}(x)=x_{1}+4 x_{3}+x_{5}-10 \leq 0, \\
& 0 \leq x_{1} \leq 12, \quad 0 \leq x_{2} \leq 18, \quad 0 \leq x_{3} \leq 5, \\
& 0 \leq x_{4} \leq 12, \quad 0 \leq x_{5} \leq 1, \quad 0 \leq x_{6} \leq 16 .
\end{array}
$$


Let $x^{0}=(3,3,3,3,1,3), q_{0}=1000, \epsilon_{0}=0.1, \eta=0.01, N=2$, numerical results by Algorithm 7 are shown in Table 3.

Table 3 Numerical results for Example 3 with $x^{0}=(3,3,3,3,1,3)$

\begin{tabular}{|c|c|c|c|c|c|}
\hline$j$ & $x_{j}^{*}$ & $q_{j}$ & $\epsilon_{j}$ & $e_{j}$ & $f\left(x_{j}^{*}\right)$ \\
\hline 0 & $\begin{array}{r}(1.657190,8.341187 \\
0.119599,0.548394 \\
0.988944,7.471706)\end{array}$ & 1000 & 0.1 & 0.002008 & 117.053718 \\
\hline 1 & $\begin{array}{r}(1.657434,8.342565 \\
0.119498,0.548084 \\
0.989852,7.472210)\end{array}$ & 2000 & 0.001 & 0.000000 & 117.071132 \\
\hline
\end{tabular}

Let $x^{0}=(4,4,4,4,1,4), q_{0}=1000, \epsilon_{0}=0.1, \eta=0.01, N=2$, the results by Algorithm 7 are shown in Table 4.

Table 4 Numerical results for Example 3 with $x^{0}=(4,4,4,4,1,4)$

\begin{tabular}{|c|c|c|c|c|c|}
\hline$j$ & $x_{j}^{*}$ & $q_{j}$ & $\epsilon_{j}$ & $e_{j}$ & $f\left(x_{j}^{*}\right)$ \\
\hline 0 & $\begin{array}{r}(1.619428,8.382967 \\
0.021739,0.600295 \\
0.997596,7.408475)\end{array}$ & 1000 & 0.1 & 0.003962 & 117.100131 \\
\hline 1 & $\begin{array}{r}(1.618086,8.381914, \\
0.022029,0.599781 \\
0.996276,7.407669) \\
\end{array}$ & 2000 & 0.001 & 0.000002 & 117.082494 \\
\hline 2 & $\begin{array}{r}(1.618086,8.381914 \\
0.022030,0.599780 \\
0.996275,7.407668)\end{array}$ & 4000 & 0.00001 & 0.000000 & 117.082487 \\
\hline
\end{tabular}

Let $x^{0}=(9,9,5,9,1,9), q_{0}=1000, \epsilon_{0}=0.1, \eta=0.01, N=2$, the results by Algorithm 7 are shown in Table 5 .

Table 5 Numerical results for Example 3 with $x^{0}=(9,9,5,9,1,9)$

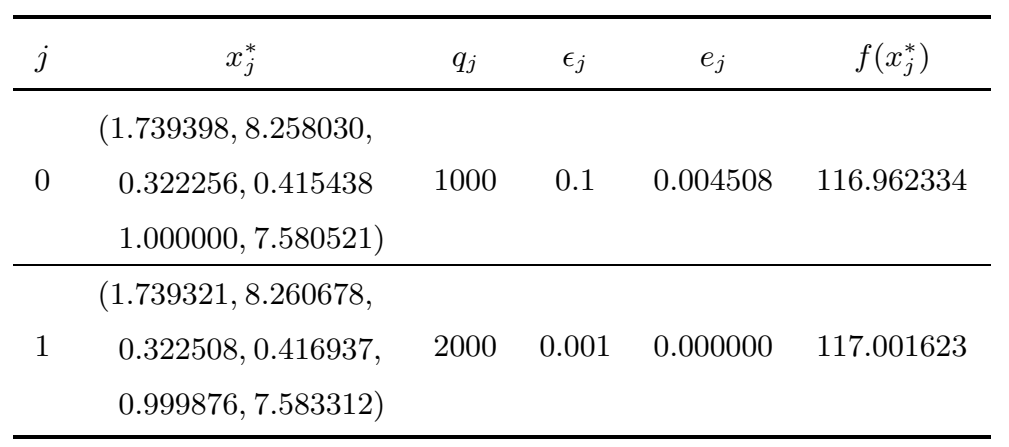


The obtained approximately optimal solution is $x^{*}=(1.739321,8.260678,0.322508,0.416937$, $0.999876,7.583312$ ) with corresponding objective function value 117.001623. From [14], the obtained approximately optimal solution is $x^{*}=(1.847052,8.152948,0.607878,0.244707,0.994467$, 7.766359) with corresponding objective function value 117.038781.

One can see that the numerical results in Table 3, Table 4 and Table 5 are similar. This means that Algorithm 7 does not completely depend on how to choose a starting point in this example.

Demonstrated by the numerical examples, Algorithm 7 is applicable for finding approximate global solutions for inequality-constrained global optimization problems.

According to our experience, initially $q_{0}$ may be $0.1,1,5,10,100,1000$ or $10000, N=2,5$, 10 or 100 , and the iteration formula $q=N q$. The initial value of $\epsilon_{0}$ may be $10,5,1,0.5$ or 0.1 , $\eta=0.5,0.1,0.05$ or 0.01 , and the iteration formula $\epsilon=\eta \epsilon$.

\section{References}

[1] Zangwill W I. Non-linear programming via penalty functions. Management Science, 1967, 13: 344-358.

[2] Bazaraa M S, Sherali H D, Shetty C M. Nonlinear programming: Theory and algorithms. 2nd ed. John Wiley Sons, Inc., New York, 1993.

[3] Bai F S, Luo X Y. Modified lower order penalty functions based on quadratic smoothing approximation. Operations Research Transactions, 2012, 16: 9-22.

[4] Lian S J. Smoothing approximation to $l_{1}$ exact penalty function for inequality constrained optimization. Applied Mathematics and Computation, 2012, 219: 3113-3121.

[5] Sun X L, Li D. Value-estimation function method for for constrained global optimization. Journal of Optimization Theory and Applications, 1999, 24: 385-409.

[6] Wang C Y, Zhao W L, Zhou J C, et al. Global convergence and finite termination of a class of smooth penalty function algorithms. Optimization Methods and Software, 2013, 28: 1-25.

[7] Xu X S, Meng Z Q, Sun J W, et al. A second-order smooth penalty function algorithm for constrained optimization problems. Computational Optimization and Applications, 2013, 55: 155-172.

[8] Yang X Q, Meng Z Q, Huang X X, et al. Smoothing nonlinear penalty functions for constrained optimization. Numerical Functional Analysis and Optimization, 2003, 24: 351-364.

[9] Yu C J, Teo K L, Zhang L S, et al. A new exact penalty function method for continuous inequality constrained optimization problems. Journal of Industrial and Management Optimization, 2010, 6: 895910.

[10] Jiang M, Shen R, Xu X, et al. Second-order smoothing objective penalty function for constrained optimization problems. Numerical Functional Analysis and Optimization, 2014, 35: 294-309.

[11] Rubinov A M, Yang X Q, Bagirov A M. Penalty functions with a small penalty parameter. Optimization Methods and Software, 2002, 17: 931-964.

[12] Huang X X, Yang X Q. Convergence analysis of a class of nonlinear penalization methods for constrained optimization via first-order necessary optimality conditions. Journal of Optimization Theory and Applications, 2003, 116: 311-332.

[13] Wu Z Y, Bai F S, Yang X Q, et al. An exact lower order penalty function and its smoothing in nonlinear programming. Optimization, 2004, 53: 51-68.

[14] Meng Z Q, Dang C Y, Yang X Q. On the smoothing of the square-root exact penalty function for inequality constrained optimization. Computational Optimization and Applications, 2006, 35: 375-398.

[15] Lian S J. Smoothing approximation to the square-order exact penalty functions for constrained optimization. Journal of Applied Mathematics, 2013, Article ID 568316, 7 pages. 